\title{
Efficient Pressure Sensors Placement for Water Distribution Network Using Flow-Tracking Analysis
}

\author{
National University of Tainan \\ W.L. Yang \\ National University of Tainan
}

Y.C. Huang ( $\square$ seaman899@yahoo.com.tw)

\section{Research Article}

Keywords: Flow-Tracking analysis, network topologies, objective function $f(X k), O(m \rrbracket n)$

Posted Date: November 19th, 2021

DOI: https://doi.org/10.21203/rs.3.rs-1061512/v1

License: (c) (i) This work is licensed under a Creative Commons Attribution 4.0 International License. Read Full License 


\title{
Efficient Pressure Sensors Placement for Water Distribution Network Using Flow-Tracking Analysis
}

\author{
Y.C. Huang ${ }^{1,{ }^{*}}$ and W. L. Yang ${ }^{2}$ \\ ${ }^{1}$ Department of Electrical Engineering, National University of Tainan, Taiwan, R.O.C. \\ ${ }^{2}$ Department of Computer Science and Information Engineering, National University of Tainan, Taiwan \\ *seman899@yahoo.com.tw
}

\begin{abstract}
This letter presents a novel approach for efficient deployment of top pressure sensors in water distribution network. FlowTracking analysis using head loss coverage ratio explores a least number of top sensors in network topologies. The following sequence of top sensor plans can be effortlessly determined by simple greedy algorithm. A regular hydraulic model with 33 sensor nodes is to validate the fast and effective feature of flow-tracking method. A top set of 5 sensor nodes selected by head loss coverage ratio $H_{\text {cr }}$ in flow-tracking analysis agree exactly with top set of 5 sensitive nodes selected by objective function $f\left(X_{\mathrm{k}}\right)$ by means of Sensitivity Analysis. A linear relationship between objective function $f\left(\mathrm{X}_{\mathrm{k}}\right)$ and heads loss coverage ratio $H_{\text {cr }}$ of top sensor nodes reveals high accuracy mapping from flow-tracking method to Sensitivity Analysis. Time complexity of searching top sensors node set by flow-tracking analysis is $O(\mathrm{~m} \times \mathrm{n})$. Average pressure error can be expected as low as $0.08 \mathrm{~m}$ with top-two sensors in sensors layout. As top sensors in deployment plan are all used, minimum error of $0.04 \mathrm{~m}$ is achieved. Flow-Tracking analysis has the advantages of little time complexity and accurate top sensors strategy as a new efficient solution for pressure sensors deployment in associated flow network.
\end{abstract}

\section{Introduction}

Pressure monitoring plays a crucial role in resources management of urban water distribution network (WDN). Pressure sensors should be located in least places where they can offer most valuable monitored data in WDN. It is unfeasible to install pressure sensors all around the network, but efficient deployment of pressure sensors using hydraulic model may assist performance insufficiency of network operation and become a viable alternative. As a project buried underground, WDN is complicated because it is composed of hundreds of thousands of pipes, junctions, pumps, valves, and storage tanks ${ }^{1}$. Thus, a hydraulic modeling (e.g. EPANET) is required as a simulation to have a comprehensive grasp on flow patterns and pressure variations of distributed network ${ }^{2}$. WDN modeling requires parameters such as water consumption, valve open/close status, and pipe roughness, etc. Water consumption and valve open/close vary with daily life and water supply operating condition. Such variables are only be monitored by instruments or mathematical predictions. But the roughness of pipes, which represent the flow resistance of pipes and fittings in the network, are parameters that will not change for a long time ${ }^{3,4}$. Moreover, pipe roughness is an important parameter for simulating the pressure distribution of the network. Therefore, the calibration of pipe roughness coefficient is important for the application of the hydraulic model ${ }^{5}$. For the calibration of the pipe roughness coefficient, the first step is to estimate the initial value of every pipe. Then compare the simulated pressure values from hydraulic model with the values from measurement ${ }^{6,7,8}$.

How to select the pressure sensor nodes in a water distribution network is critical to the pipe roughness calibration. Schaetzen et al. presented three methods to select pressure sensor nodes to calibrate pipe roughness coefficients, one of which rank the sampling locations based on shortest path algorithms ${ }^{9}$. Klapcsik et al. discussed two approaches to tackle the problem of locating the top pressure sensor nodes in a hydraulic system ${ }^{10}$. One of them applies the concept borrowed from graph theory to optimize the pressure measuring locations, and the other applies the sensitivity analysis of the pipe roughness affecting the node pressure. However, the above methods lack the physical bases for the fluid dynamics of water supply. Yoo et al. developed a method considering the pipe connectivity of water distributed network and the impact among nodes by pressure driven analysis and entropy method ${ }^{11}$. Lee et al. defined a concept of coverage, and proposed methods on how to locate monitoring sites by an analysis of the pathways of water flows in a designated water network ${ }^{12}$. Even though Yoo and Lee's works are connected to the physical properties of the pipe network, both of them do not grasp the pressure change of entire loop from the water source to the pressure sensor node.

This letter proposes a new approach, relying on coverage concept of head loss in flow tree diagram, to select the top location set for pressure sensors deployment efficiently. ${ }^{12}$ According to the energy conservation law of fluid dynamics, their relationships are developed by means of the flow tracking analysis from water source to pressure sensor node. Sequence configuration for these selected top sensor nodes group is easily constructed by greedy algorithm, and hence improving the computational efficiency significantly. 


\section{Methods}

Water head refers to the pressure of a pipe water at the node (expressed in meters of water), plus the elevation of the node (expressed in meters). Therefore, unit for pressure and water head used in this paper is meter. For convenience, the term pressure can replace water head if the elevation of the node is zero. The head loss of pipe is the pressure drop caused by the surface friction inside the pipe. Head loss in the pipe can be calculated with the Hazen-Williams formula, which is known as empirical equation frequently for evaluation of pressure drop in water distribution networks ${ }^{2}$. Head loss $\left(\boldsymbol{h}_{\boldsymbol{L}}\right)$ is determined by flow rate $(\boldsymbol{q})$, pipe roughness coefficient $(\boldsymbol{C})$, pipe diameter $(\boldsymbol{d})$, and pipe length $(\boldsymbol{L})$ as expressed in following HazenWilliams equation,

$$
\boldsymbol{h}_{L}=\boldsymbol{A} \boldsymbol{q}^{1.852}
$$

where

$$
\boldsymbol{A}=4.727 \boldsymbol{C}^{-1.852} \boldsymbol{d}^{-4.871} \boldsymbol{L}
$$

A network $W(R, V, A)$ as shown in Fig. 2, where $R$ is the set of water sources, $V$ is the set of nodes and $A$ is the set of edges (or pipes) in the network. The water head (or pressure) at node $i \in V$, can be calculated by the following hydraulic model formula.

$$
P_{i}=f\left(H R_{j}, H L_{k}\right)
$$

where $P_{i}$ is the simulated pressure at $i^{\text {th }}$ node, $1 \leq i \leq|V| .|V|$ is the number of nodes. $H R_{j}$ is the set of all water heads (or pressures) of water sources, $1 \leq j \leq|R|$, and $H L_{k}$ is the set of all head losses of the pipes, $1 \leq k \leq|A| .|R|$ and $|A|$ are the number of water sources and the number of pipes, respectively. If $H R_{j}$ is known and $C_{k}$ represents the roughness coefficient of $k^{\text {th }}$ pipe in Eq. (2), we can compute the head loss $h_{L}$ for all the pipes. As a result, the pressure at each node $P_{i}$ is then obtained by Eq. (2), $1 \leq i \leq$ $|V|$. In the practical network operations, it is often assumed that the values of $q, d$, and $L$ in Eq. (1) are given. Hence, the problem of given $H L_{k}$ set is corresponding to the problem of getting appropriate $C_{k}$. Where $C_{k}$ is the set of all pipe roughness coefficients, $1 \leq k \leq|A|$. To avoid random speculation of $H L_{\mathrm{k}}$, a node subset $U(U \subseteq V,|U|=m)$, installed with pressure sensors is necessary. An typical hydraulic model with two water sources, 33 nodes, and 50 pipes was adopted as shown in Fig. 1. All node elevations are set to be zero for simplicity. The red lines denote the main pipes whose diameters are $200 \mathrm{~mm}$, the diameters of the other pipes are $100 \mathrm{~mm}$, and the length of each pipe is $1000 \mathrm{~m}$. All nodal demands are set to be $4.0 \mathrm{~m}^{3} / \mathrm{h}$ and the supply pressure head of both water sources are $35 \mathrm{~m}$.

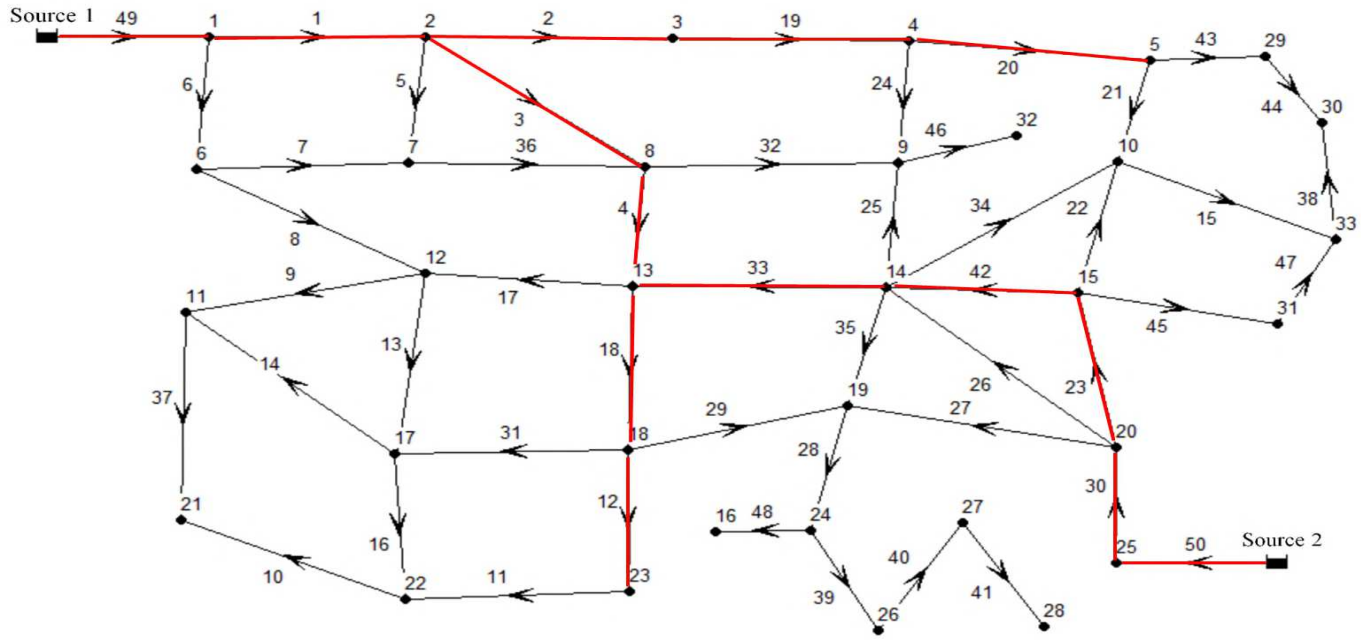

Figure 1. A typical hydraulic model with flow direction.

A number of methods for optimal location of pressure sensors in earlier works were introduced. The following will describe in detail how to use flow-tacking analysis to find optimal sensor nodes. According to fluid dynamics, headloss coverage ratio $\boldsymbol{H}_{\boldsymbol{c} r}$ is defined as

$$
\boldsymbol{H}_{\boldsymbol{c r}}=\frac{\sum_{i=1}^{M} h_{j}^{*}}{\sum_{i=1}^{N} h_{i}}
$$


where $N$ and $M$ are total number of pipes in the network and on the flow-tracks with measuring nodes, respectively. $h_{j}^{*}$ represents the head loss of the $j^{\text {th }}$ pipe in those flow-tracks. $\boldsymbol{H}_{\boldsymbol{c} r}$ is a fraction of coverage desired to be unity, as indicating the preferred sensor nodes can cover total pipes loss in network entirely.

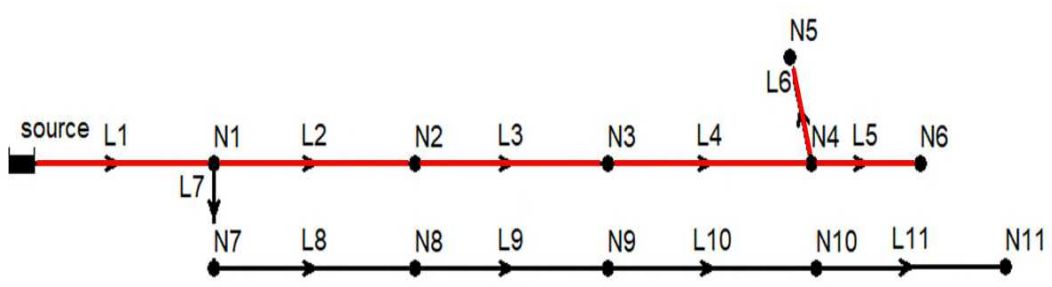

(a)

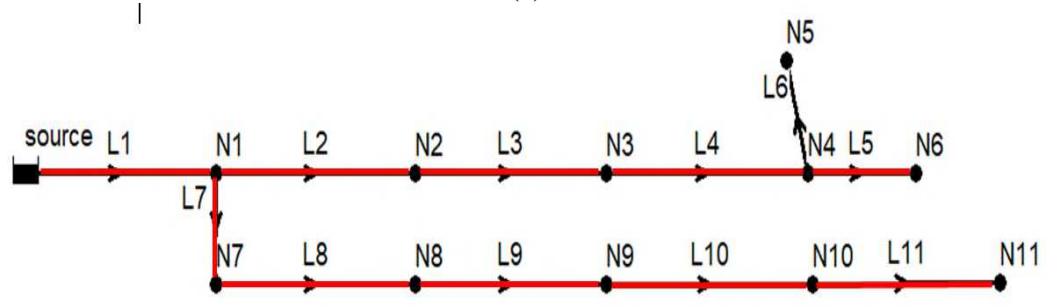

(b)

Figure 2. Flow-Tracking of end nodes (a) N5-N6 and (b) N6-N11 in two branches in water distribution system

Figure 2 shows a typical two branches on the water distribution system with three end nodes of N5, N6, and N11. All the pipes in Fig. 2 have same roughness coefficient, diameter, and length. However, if two pressure sensors can only be installed, two options N11-N5 and N11-N6 are better than N5-N6. Since more pipes are covered by first two options than by the third option. The $\boldsymbol{H}_{c r}$ of N6-N11 is 0.994 higher than 0.739 of N5-N6. It means that N6-N11 is a better choice than N5-N6 mostly for the reason that $N 11$ is a major end node as mentioned above. Therefore, this study will begin a procedure by evaluating $\boldsymbol{H}_{c r}$ as the index of priority rank in the greedy algorithm as follows. Steps (1)-(7) are flow-tracking analysis and greedy algorithm proceeds as described:

(1) Calculate flow directions of all pipes with EPANET. The pipe connects two nodes, and water flows from the upstream node to the downstream node.

(2) Search for nodes where water flow can only enter and no out. These sensor nodes are candidates for installing pressure sensors.

(3) Calculate $\boldsymbol{H}_{c r}$ for each sensor node.

(4) Sort all the $\boldsymbol{H}_{c r}$ values in descending order.

(5) The node with the largest $\boldsymbol{H}_{c r}$ is the first priority sensor node.

(6) In addition to the maximum $\boldsymbol{H}_{c r}$, select another sensor node among the other sensor nodes, so that the combination $H_{c r}$ of the two nodes is maximized. This is the second priority sensor node.

(7) With the same routine as step (6), the third, fourth, and until the last priority sensors all get into positons.

\section{Results and Discussion}

Five top sensor nodes in Fig. 3 are determined according to step (2). The flow-tracking of node 21 is shown in Fig. 4. The $H_{c r}$ of node 21 is calculated from pipes with red color. The $\boldsymbol{H}_{c r}$ of five sensor nodes is shown in Table 1. Column I in Table 1 is the sensor nodes sorted by $\boldsymbol{H}_{c r}$ in descending order. Table 1 is the result of flow-tracking method. The next stage is continued by greedy algorithm. Since node 28 is the first priority, all combinations of the other nodes with node 28 are shown in column III in Table 2. Since $\boldsymbol{H}_{c r}$ of node 28 and 30 is the highest in column IV, node 30 is the second priority of the list. It can be seen from Table 1 that $\boldsymbol{H}_{c r}$ of node 16 is ranked second, but its jointed result with node 28 is far inferior to the jointed result of node 30 and node 28. After greedy algorithm, node 16's final selection ranking fell to fifth. The reason is obvious from Fig. 5 that node 16 and 28 are only one pipe (pipe 48 ) in difference. 


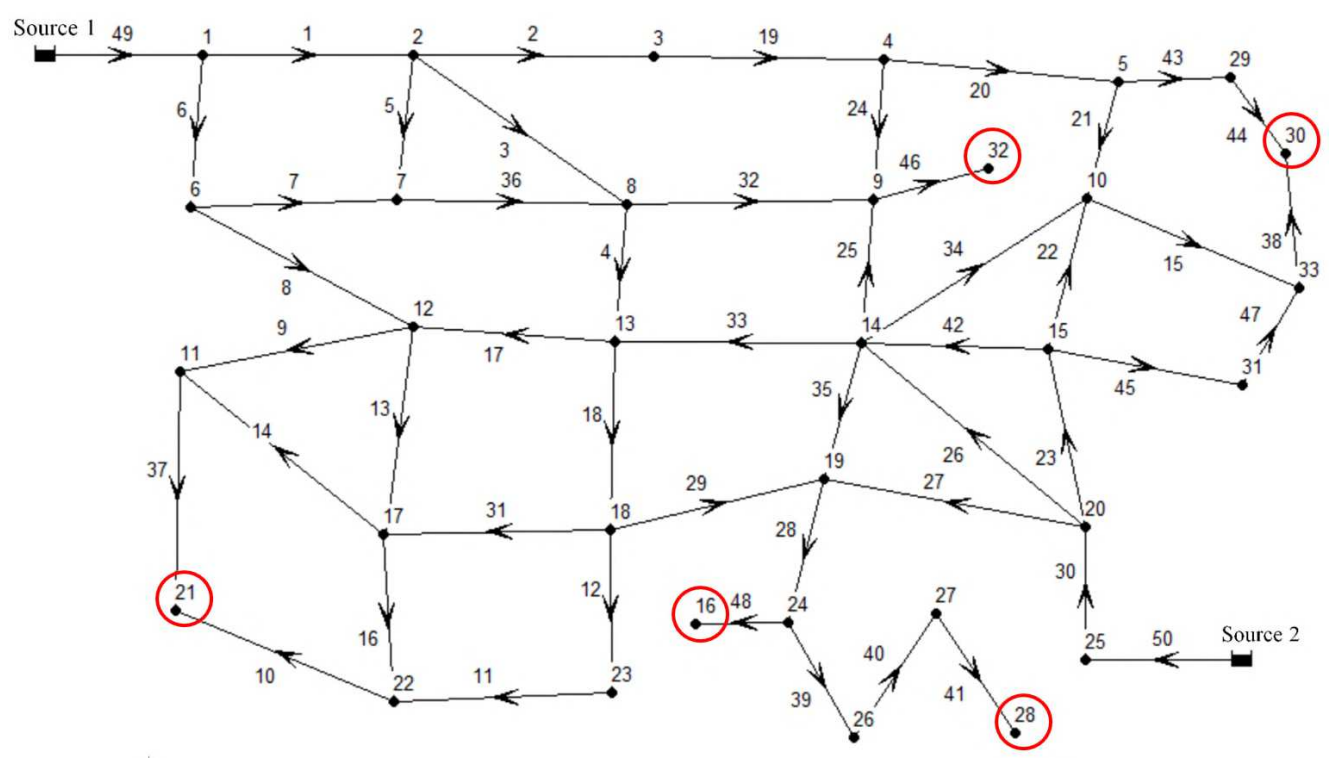

Figure 3. Five optimal sensor nodes marked as red circle

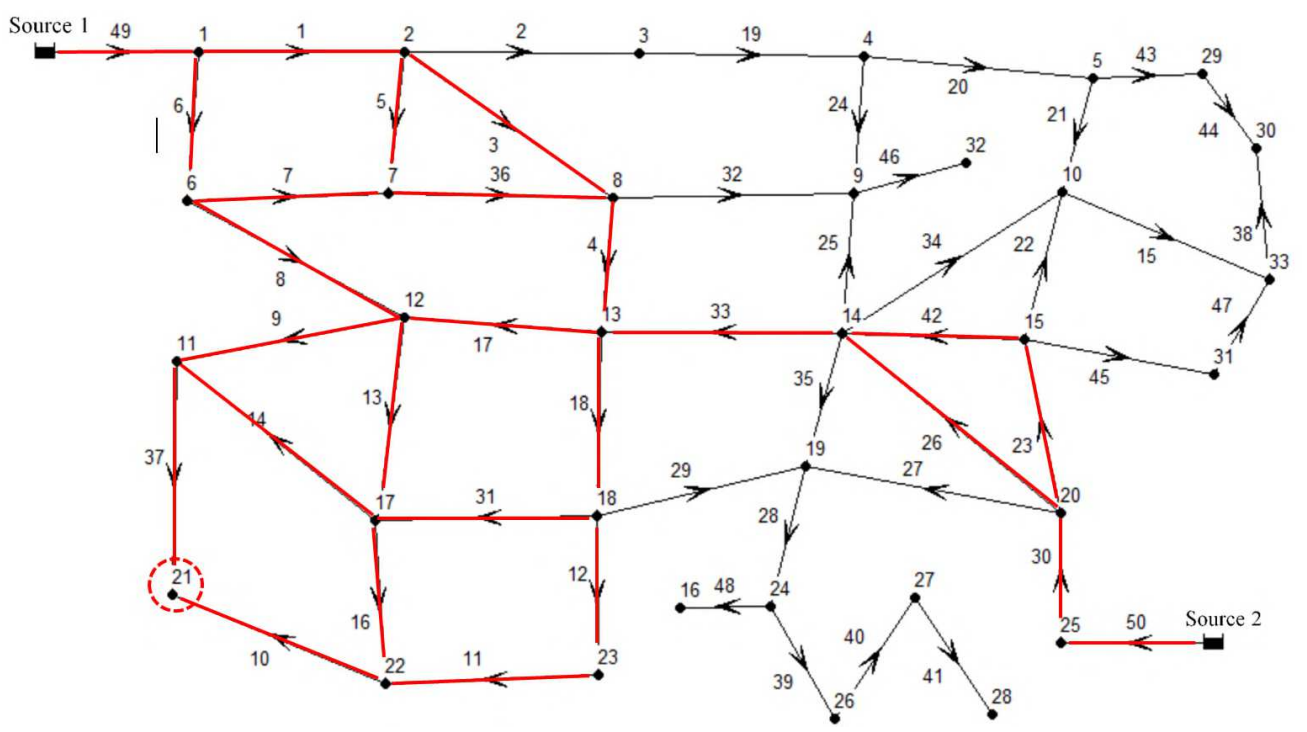

Figure 4. Flow-Tracking red tree diagram of node 21

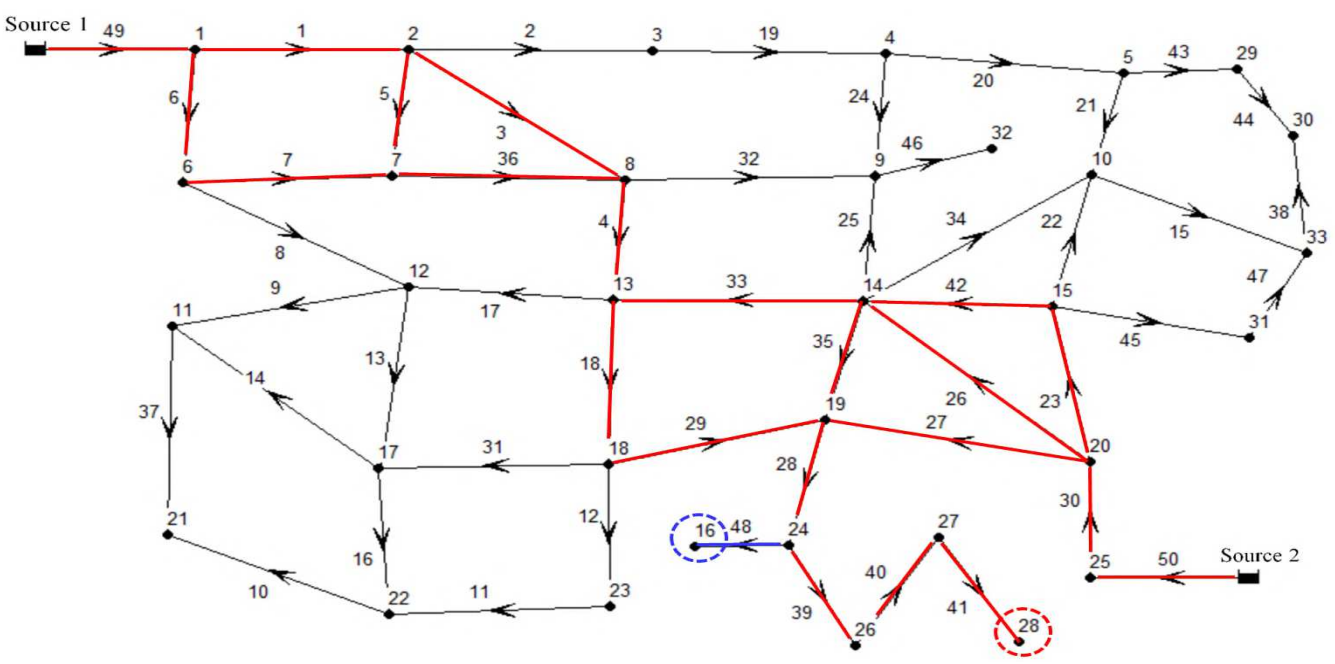

Figure 5. Flow-Tracking red tree diagram of node 28 and 16. 
Table 1. $H_{c r}$ and FTG rank of optimal sensor nodes by flow-tracking method

\begin{tabular}{|c|c|c|}
\hline Node & $\boldsymbol{H}_{c r}$ & FTG rank \\
\hline 28 & 0.758 & 1 \\
\hline 16 & 0.632 & 5 \\
\hline 21 & 0.373 & 3 \\
\hline 32 & 0.297 & 4 \\
\hline 30 & 0.271 & 2 \\
\hline
\end{tabular}

Table 2. Rank of grouped sensor nodes by Greedy algorithm

\begin{tabular}{|c|c|c|c|c|c|c|c|c|c|}
\hline $1^{\text {st }}$ node & $H_{c r}$ & $\begin{array}{l}1^{\text {st }}-2^{\text {nd }} \\
\text { nodes }\end{array}$ & $H_{c r}$ & $\begin{array}{c}1^{\text {st }}-2^{\text {nd }}-3^{\text {rd }} \\
\text { nodes }\end{array}$ & $H_{c r}$ & $\begin{array}{c}1^{\text {st }}-2^{\text {nd }}-3^{\text {rd }}-4^{\text {th }} \\
\text { nodes }\end{array}$ & $H_{c r}$ & $\begin{array}{c}1^{\text {st }}-2^{\text {nd }}-3^{\text {rd }}-4^{\text {th }}-5^{\text {th }} \\
\text { nodes }\end{array}$ & $H_{c r}$ \\
\hline 28 & 0.758 & $28-30$ & 0.863 & $28-30-21$ & 0.962 & $28-30-21-32$ & 0.989 & $28-30-21-32-16$ & 1.000 \\
\hline 16 & 0.632 & $28-21$ & 0.857 & 28-30-32 & 0.890 & $28-30-21-16$ & 0.973 & & \\
\hline 21 & 0.373 & $28-32$ & 0.800 & 28-30-16 & 0.874 & & & & \\
\hline 32 & 0.297 & $28-16$ & 0.769 & & & & & & \\
\hline 30 & 0.271 & & & & & & & & \\
\hline
\end{tabular}

\section{High Compatibility with Sensitivity Analysis}

Just like flow-tracking analysis trying to find the pressure sensors deployment by fluid dynamics, Sensitivity analysis (SA) uses an intuitive method to search the most sensitive pressure sensor point ${ }^{9,10}$. In order to find the best combination of $k$ sensor nodes, Eq. (4) is proposed by Klapcsik et al. ${ }^{10}$.

$$
f\left(\mathbf{X}_{k}\right)=\sqrt{\sum_{i=1}^{2}\left[\frac{F_{i}\left(\mathbf{X}_{k}\right)-F_{i, \max }}{F_{i, \text { min }}-F_{i, \max }}\right]^{2} w_{i}}
$$

where $w_{i}$ is the weight of $\boldsymbol{F}_{\boldsymbol{I}}\left(\mathrm{X}_{\mathrm{k}}\right)$ and $\boldsymbol{F}_{2}\left(\mathrm{X}_{\mathrm{k}}\right), \boldsymbol{F}_{2, \max }=\ln (N)$ and $N$ is the total number of pipes. The objective is to define the sampling set that has the minimum values of Eq. (1). Obviously, the flow-tracking analysis (FTA) and sensitivity analysis (SA) use different index, $\boldsymbol{H}_{\mathrm{cr}}$ and $\boldsymbol{f}\left(\mathrm{X}_{\mathrm{k}}\right)$ as the parameters for selecting the sensor nodes. However, no matter which possible order among the optimal set would be, optimal set of sensor nodes by both FTA and SA are exactly the same (Table 3). In order to verify this unique relationship, $f\left(\mathrm{X}_{\mathrm{k}}\right)$ in Table 3 shows an excellent matching in each possible combination. Flow-Tracking analysis demonstrates a great agreement and perfect link directly to Sensitivity analysis.

Head loss has exponential power sensitivity to roughness coefficient as in Eq. (1). In other words, head loss $\left(\boldsymbol{H}_{\mathbf{c r}}\right)$ and sensitivity $\boldsymbol{f}\left(\mathrm{X}_{\mathrm{k}}\right)$ reached at one goal. $\boldsymbol{f}\left(\mathrm{X}_{\mathrm{k}}\right)$ is a search strategy function in SA and $\boldsymbol{H}_{\mathrm{cr}}$ is the flow-tracking principle behind search strategy of SA. Figure 6 reveals a linear correlation between $\boldsymbol{f}\left(\mathrm{X}_{\mathrm{k}}\right)$ and $\boldsymbol{H}_{\mathrm{cr}}$ in optimal combination of top sensors. The correlation coefficient $r$ closes to -1 indicating that a perfect linear relationship is discovered.

Table 3. Comparison of $\boldsymbol{f}\left(\mathrm{X}_{k}\right)$ in Sensitivity analysis, $\boldsymbol{H}_{c r}$ in Flow-Tracking analysis, and $P_{\text {err }}$ of optimal set.

\begin{tabular}{|l|c|c|c|}
\hline \multicolumn{1}{|c|}{ Node } & $f\left(X_{k}\right)$ & $H_{c r}$ & $P_{\text {err }}$ \\
\hline 28 & 0.378 & 0.758 & 0.196 \\
\hline 28,30 & 0.324 & 0.863 & 0.078 \\
\hline $28,30,21$ & 0.285 & 0.962 & 0.065 \\
\hline $28,30,21,32$ & 0.267 & 0.989 & 0.058 \\
\hline $28,30,21,32,16$ & 0.255 & 1.000 & 0.041 \\
\hline
\end{tabular}




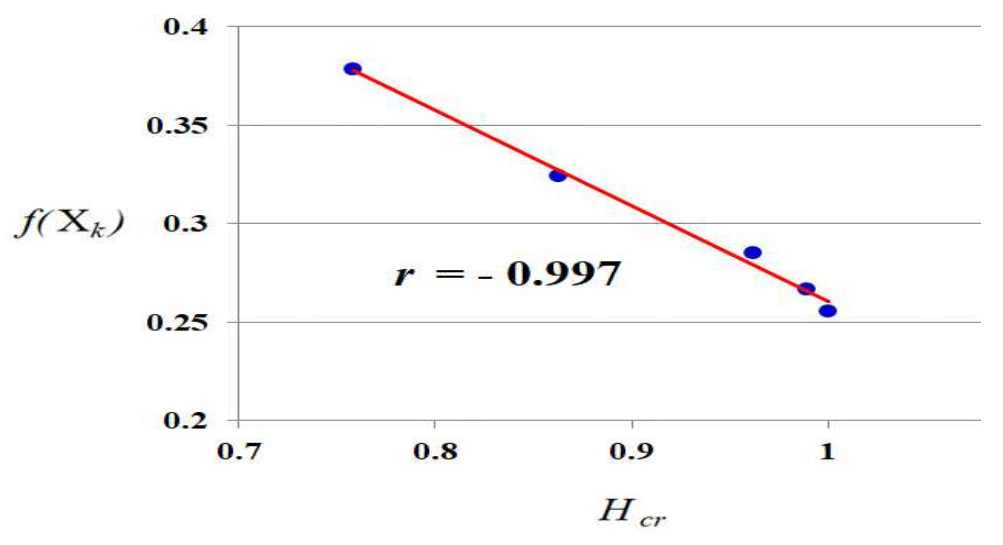

Figure 6. A perfect linear correlation between $\boldsymbol{f}\left(\mathrm{X}_{\mathrm{k}}\right)$ and $\boldsymbol{H}_{c r}$ in best combination of optimal sensors.

A major difference in solving problem of pressure sensor deployment between Sensitivity analysis and Flow-Tracking analysis is their efficiency. Equation (5) is the time complexity of SA. Therefor the time complexity of SA is the number of combinations of $\mathrm{m}$ sensor nodes selected from $\mathrm{n}$ network nodes.

$$
T(n, m)=O\left(C_{n}^{m}\right)=n ! /(m ! *(n-m) !)
$$

Time complexity regarding algorithm is generally expressed in terms of big $O$ notation, which describes operation time run an algorithm taken for performance evaluation. The time complexity $T(n)$ of the FTA depends on the structure of the network,

$$
\begin{aligned}
T(n, m) & =O(m \times n) & & \text { if } m \times n \geqq m ! \\
& =O(m !) & & \text { if } m !>n \times m
\end{aligned}
$$

Where $n$ is the number of all nodes and $m$ is the number of sensors. For a huge network but less sensors $(n>m)$, degree of complexity depends on the flow-tracking method in step (3). But in the case of more sensor nodes, the size of complexity turns to operation of the greedy algorithm only. Because SA lacks the theoretical guidance for selecting sensor nodes, it can only choose randomly and compare the value of Eq. (6). The ratio of time complexity in choosing optimal 5 sensor nodes out of 33 nodes by Eq. (6) of FTA to Eq. (5) of SA is $33 \times 5=165$ to $C_{33}^{5}=237336$.

The average pressure simulated error $\boldsymbol{P}_{\text {err }}$ will improve the reliability.

$$
\boldsymbol{P}_{\text {err }}=\frac{\sum_{j=1}^{T_{n}} E_{d j}}{T_{n}}
$$

Where $E_{d j}$ is pressure error of the $j^{\text {th }}$ test and $T_{n}$ is the total test number set to 100 in this paper. The $P_{e r r}$ of top rank combination in optimal set is shown in Table 3. $P_{\text {err }}$ less than $0.1 \mathrm{~m}$ can satisfy the error requirement of the hydraulic model calibration. In this case, FTA needs only two sensor nodes 28 and 30 for the model verification.

In the future, moreover, FTA can make the work of pressure sensor deployment in massive water distribution network much easier by partitioning WDN into several smaller sub-networks. Then pressure sensor deployment in sub-networks can be resolved one by one with FTA algorithm. Optima sensors node set in each sub-network can be integrated, and successive execution using FTA becomes faster and simpler. The resulting deployment process for $\mathrm{P}$ in enormous complicated WDN by means of FTA saves more time and work. Consequently, FTA highlighting fast and little time complexity is especially valuable to offer optimal top sensors set of the deployment layout efficiently and cost-effectively.

\section{References}

1. Novak, P., Guinot, V., Jeffrey, A., and Reeve, D. E., Hydraulic Modelling - an Introduction: Principles, Methods and Applications, Spon Press, New York, USA, (2010).

2. Rossman, L.A., EPANET 2 User's Manual, U.S. Environmental Protection Agency, Washington, D.C., EPA/600/R00/057, (2000).

3. Jeppson, R. W., Steady Flow Analysis of Pipe Networks: An Instructional Manual, (1974). 
4. Lu, H. Y., Wang, J., and Yuan, Y., "A New Method for Calibrating Microcosmic Model of Municipal Water Distribution Network," 2010 International Symposium on Computational Intelligence and Design, pp. 117-119, (2010).

5. Ormsbee, L. E., and Lingireddy, S., "Calibration of Hydraulic Network Models," Journal-American Water Works Association, vol. 89, no. 2, pp. 42-50, (1997).

6. Do, N., Simpson A., Deuerlein, J., and Piller, "Calibration of Water Demand Multipliers in Water Distribution Systems Using Genetic Algorithms," J. Water Resour. Plann. Manage. vol. 142, no. 11, 04016044, (2016).

7. Ostfeld, A., et al. "The Battle of Water Calibration Networks (BWCN)." J. Water Res. Plan. Manage., vol. 138, no. 5, pp. 523-532, (2012).

8. Lingireddy, S. and Ormsbee, L.E., "Hydraulic Network Calibration Using Genetic Optimization," Civ. Eng. Environ. Syst., vol. 19, no. 1, pp. 13-39, (2002).

9. de Schaetzen, W. B. F., Walters, G. A., and Savic, D. A., “Optimal Sampling Design for Model Calibration Using Shortest Path, Genetic and Entropy Algorithms," Urban Water, vol. 2, no. 2, pp. 141-152, (2000).

10. Klapcsik K., Varga, R., and Hős, C., “Optimal Pressure Measurement Layout Design in Water Distribution Network Systems," Periodica Polytechnica Mechanical Engineering, vol. 62, no. 1, pp. 51-64., (2018).

11. Yoo, D., Chang D., Song,,Y., and Lee, J., “Optimal Placement of Pressure Gauges for Water Distribution Networks Using Entropy Theory Based on Pressure Dependent Hydraulic Simulation,” Entropy, vol. 20, no. 8, pp. 576-599, (2018).

12. Lee, B. H. and Deininger R. A., "Optimal Locations of Monitoring Stations in Water Distribution System," J. Environmental Engineering, vol. 118, no. 1, pp. 4-16, (1992). 

